\title{
Development of a Patient-Reported Outcome Measure for Non-Alcoholic Steatohepatitis (NASH-CHECK): Results of a Qualitative Study
}

\author{
Lynda C. Doward ${ }^{1} \cdot$ Maria-Magdalena Balp ${ }^{2}$ (1) James Twiss ${ }^{1} \cdot$ Christina Slota $^{3} \cdot$ Donna Cryer $^{4} \cdot$ Clifford A. Brass $^{5}$. \\ Quentin M. Anstee $^{6,7} \cdot$ Arun J. Sanyal ${ }^{8}$
}

Accepted: 18 November 2020 / Published online: 18 December 2020

(c) The Author(s) 2020

\begin{abstract}
Background Non-alcoholic steatohepatitis (NASH) is a progressive form of non-alcoholic fatty liver disease characterized by excessive liver fat accumulation, inflammation, cell injury, and fibrosis. It is viewed as largely asymptomatic in its earlier (non-cirrhotic) stages, and information on the patient-perceived impact of NASH is scarce.

Objective This study aimed to develop a NASH-specific patient-reported outcome (PRO) measure (NASH-CHECK) for use as a trial endpoint, using methods compliant with regulatory expectations.

Methods A NASH conceptual model was developed based on the literature and clinical/patient expert review. The model guided concept elicitation (CE) interviews in patients with non-cirrhotic NASH recruited via a US tertiary care center. NASH-CHECK content was generated via thematic analysis of CE data and review by clinical/patient experts. Cognitive debriefing (CD) interviews with US patients evaluated content validity.

Results The literature review confirmed that NASH impacts on functioning and health-related quality of life (HRQoL). Overall, $23 \mathrm{CE}$ and $20 \mathrm{CD}$ interviews were conducted. Key symptoms reported in CE interviews included pain in the upper-right abdomen $(n=14)$, fatigue $(n=18)$, poor sleep quality $(n=12)$, impaired memory $(n=13)$, and reduced focus $(n=11)$; key HRQoL impacts included impaired physical functioning, reduced ability to conduct daily living tasks, reduced quality of relationships, low mood, anxiety, and self-consciousness. The 52-item first-draft NASH-CHECK was reduced to 31 items based on patient feedback on item relevance, acceptability, and comprehension.

Conclusions The interviews revealed key symptoms and broad HRQoL impacts of NASH. As a disease-specific PRO measure assessing symptoms and HRQoL, the NASH-CHECK is relevant, comprehensive, and acceptable to patients and clinicians.
\end{abstract}

\section{Introduction}

Non-alcoholic fatty liver disease (NAFLD), the most common chronic liver disease, is characterized by a build-up of fat in the liver in the absence of excessive alcohol consumption [1-3]. NAFLD occurs in the context of metabolic syndrome, being prevalent among patients with type 2 diabetes mellitus, obesity, and cardiovascular diseases [4, 5]. Approximately $25-30 \%$ of patients with NAFLD will develop non-alcoholic steatohepatitis (NASH), the progressive and most severe form of NAFLD [6]. Progression to NASH is characterized by inflammation of the liver, with

Quentin M. Anstee and Arun J. Sanyal joint senior authors.

Lynda C. Doward

ldoward@rti.org

Extended author information available on the last page of the article evidence of liver cell injury and different degrees of scarring or fibrosis $[7,8]$. NASH can lead to progressive liver cirrhosis and eventual liver failure, with associated morbidity and mortality $[9,10]$.

NASH is often considered an asymptomatic, "silent" disease in its early stages; however, symptoms such as chronic fatigue, malaise, apparent hepatomegaly, and upper-right quadrant abdominal fullness and discomfort have been reported $[3,11,12]$. Because the condition is viewed as largely asymptomatic, published literature on the patientperceived impact of NASH has been scarce, although recent evidence suggests that patients with NASH experience symptom burden and impaired health-related quality of life (HRQoL) [13-17]. Histologic surrogate endpoints (such as reduction of hepatic fibrosis, inflammation, and hepatocellular ballooning) or non-invasive measures (magnetic resonance-measured hepatic fat content and serologic biomarkers for early phase trials) are most often included as 


\section{Key Points for Decision Makers}

Non-alcoholic steatohepatitis (NASH) is often considered an asymptomatic, "silent" disease in its early stages, but evidence suggests that patients experience symptoms, including fatigue, malaise, and abdominal discomfort, that can impair health-related quality of life (HRQoL).

As reported in qualitative interviews with patients, symptoms of non-cirrhotic NASH include abdominal pain, fatigue, poor sleep, and cognition problems. NASH affects patients' physical, social, and emotional functioning and ability to perform daily activities.

A new patient-reported outcome measure of NASH symptoms and HRQoL, the NASH-CHECK has been developed. The NASH-CHECK is relevant, comprehensive, acceptable to patients, and suitable for use in clinical trials and clinical settings to evaluate patientperceived impact of NASH.

primary endpoints in clinical trials in NASH [18-20]. While these endpoints present crucial information on the histological manifestations of the disease to clinicians, they do not reflect the impact of the disease and the treatment outcomes that matter most to patients.

Patient-reported outcome (PRO) measures (PROMs) are standardized means of evaluating symptoms, functioning, HRQoL, and the wider impact of a disease from the patient perspective. PROs add value by complementing key clinical endpoints in clinical trials [21]. Such information is vital in characterizing patients' views on the effectiveness of an intervention. In addition, PROMs are useful for monitoring patients in clinical practice, allowing physicians to evaluate patients' symptom experiences and changes with treatment alongside physician-rated assessments [21]. PRO data are viewed as important by major regulatory authorities to capture the patient perspective in the drug development process [22]. As such, the US FDA has provided guidance for evaluation of PROMs intended for label claims, and the European Medicines Agency (EMA) has issued a reflection paper on the use of HRQoL measures in the drug evaluation process $[23,24]$. The quality standards outlined therein emphasize the need for input from the specific target population during development of a PROM and evidence of content validity based on cognitive debriefing (CD) interviews [23, 24]. However, few of the currently available PROMs for NASH meet these criteria [25].

The objective of this study was twofold: (1) to understand the humanistic burden and day-to-day impact of living with non-cirrhotic NASH and (2) to use this information to develop a novel PROM to assess symptoms and HRQoLrelated impacts of NASH that aligns with regulatory guidance. The measure will facilitate understanding of the symptoms and wider impact of NASH from the patient perspective and will provide a valuable complement to support key clinical endpoints in NASH.

\section{Materials and Methods}

An international NASH PRO Task Force comprising PRO researchers, patient advocacy representatives, clinical experts, and representatives of the pharmaceutical industry was established to explore the humanistic burden of NASH and to oversee the development of the NASH-CHECK PROM. Institutional review board approval was granted for the study by the Western Institutional Review Board (Puyallup, Washington).

Development of the NASH-CHECK PROM followed FDA guidance for PROMs intended for use in regulatory label claims and also was consistent with the EMA's reflection paper on HRQoL measures [22-24]. The FDA guidance recommends that the development of a PROM should be based on qualitative research conducted with the target population to establish the content validity of the measure. The measure should then undergo a quantitative (psychometric) evaluation to explore its measurement properties [23]. Qualitative development of the NASH-CHECK, consistent with FDA guidance, included three phases to establish the content validity of the measure: (1) development of a draft conceptual model based on literature search and discussions with medical experts; (2) content generation through concept elicitation (CE) interviews with patients diagnosed with NASH and fibrosis levels F1-F3 based on the draft conceptual model; and (3) evaluation of content validity through $\mathrm{CD}$ interviews with the same target population [23]. Specifically, the CE interviews explored the patient-perceived impact of living with NASH and informed item generation. The CD interviews evaluated the appropriateness and comprehensibility of the measure. Future planned analyses will explore the psychometric properties of the measure.

\subsection{Literature Review}

A targeted literature review was conducted using MEDLINE and Embase to identify articles published between 1996 and 2016 relating to the symptomatic and humanistic burden of non-cirrhotic NASH. Additionally, the review sought to identify validated PROMs developed specifically for this patient population. The targeted review was supplemented by a manual search of gray literature (general internet searches and congress websites), patient blogs, and patient group websites, including the British Liver Trust and the 
Global Liver Institute, to search for qualitative information on patients' experiences of living with non-cirrhotic NASH $[26,27]$. Areas of patient-reported symptoms and impact on daily life and HRQoL associated with NASH from the published and gray literature searches and social media sites were summarized and reviewed with clinical experts and patient advocacy representatives. These results were used to develop an initial conceptual model for NASH

\subsection{Study Participants}

Patients were recruited in the USA from a tertiary care center in Richmond, Virginia, and the Texas Liver Institute, San Antonio, Texas. A purposive sampling approach was used to achieve a sample size sufficient to support qualitative analyses and concept saturation [28]. For this study, non-cirrhotic patients were targeted, since the common and often severe impairments associated with cirrhosis of any cause have the potential to overwhelm any HRQoL findings that may be specific to NASH.

The inclusion criteria were adults aged 18-75 years with liver biopsy-confirmed NASH with fibrosis levels F1, F2, or F3 based on the NASH Clinical Research Network histological scoring system [29] or phenotypic diagnosis (i.e., alanine transaminase levels $[\geq 60 \mathrm{IU} / \mathrm{L}$ for males or $\geq 40 \mathrm{IU} / \mathrm{L}$ for females] and body mass index $\left[\mathrm{kg} / \mathrm{m}^{2}\right][\geq 23$ in Asian individuals or $\geq 27$ in non-Asian individuals]) and type 2 diabetes mellitus. Patient recruitment from the Texas Liver Institute had the additional inclusion criterion of Hispanic ethnicity.

The exclusion criteria were patients with other liver disease, other serious comorbid conditions, clinical evidence of cirrhosis, excessive alcohol consumption (defined as more than $20 \mathrm{~g}$ per day in females and more than $30 \mathrm{~g}$ per day in males, on average, for a period of more than 3 consecutive months within 1 year prior to the study), active substance use, severe mental illness, extreme obesity, or poorly controlled diabetes (glycated hemoglobin [HbA1c] levels $\geq 9.5 \%[80 \mathrm{mmol} / \mathrm{mol}])$.

\subsection{Elicitation of Patient-Reported Symptoms and Impacts of Non-Alcoholic Steatohepatitis (NASH)}

\subsubsection{Concept Elicitation Interviews}

Each qualitative $\mathrm{CE}$ interview with the eligible NASH patients was led by two experienced qualitative researchers and followed a semi-structured interview guide based on the draft conceptual model. The interviews explored patient experiences of NASH symptoms and the impact of NASH on the way patients feel and function. All interviews were conducted in person at a facility close to the recruiting center and were audio recorded and transcribed. Each CE interview lasted approximately $1 \mathrm{~h}$. Patient sociodemographic data were obtained from a patient-completed background questionnaire and medical data from patient records. Electronic Supplementary Material (ESM) 1 presents additional details about the CE interview methodology.

\subsubsection{Concept Elicitation Analyses}

Data analysis of the anonymized CE interview transcripts and the interviewers' field notes were performed using ATLAS.ti qualitative analysis software (ATLAS.ti Scientific Software Development GmbH; Berlin, Germany). Thematic analysis was conducted by two independent researchers to evaluate key areas of symptomatic and HRQoL impact from the patient perspective [30]. An initial coding frame-comprising topic areas, concepts, and specific symptoms and impacts - was developed based on the interview guide. This coding frame was applied to the data by assigning codes to segments of text in the interview transcripts. Interview transcripts were analyzed in sets of five. The coding frame was modified progressively to accommodate information arising from the analysis. Thematic saturation was reached when no new symptoms or impacts were identified during successive transcript sets. The size of the sample was driven by thematic saturation [28]. Thematic analysis of the data from the $\mathrm{CE}$ interviews was used to derive the final conceptual model.

\subsection{NASH-CHECK Development}

The NASH-CHECK PROM was developed based on the conceptual model for NASH. Item generation was guided by the conceptual model and the qualitative data from the $\mathrm{CE}$ interviews. An initial item pool was presented to the NASH PRO Task Force, during which the symptoms and HRQoL impacts identified were reviewed and the draft item set was selected. The Task Force also agreed on the instructions, question format, response options, and recall period for the draft measure.

\subsection{Content Validation of the NASH-CHECK Measure}

\subsubsection{Cognitive Debriefing Interviews}

Content validation was evaluated through three rounds of $\mathrm{CD}$ interviews with NASH-diagnosed patients according to the inclusion and exclusion criteria. The first round of interviews was designed to identify any initial problems with wording of instructions, items, and response options and to select between alternative formulations of individual items. The second and third rounds were intended to assess the adequacy of changes made to the measure. The purpose of all interviews was also to assess the comprehensibility, 
understandability, and relevance of the instructions, items, and response options for the NASH-CHECK. Each interview was conducted by two PRO researchers with expertise in qualitative research using a semi-structured interview guide. Rounds 1 and 2 of the interviews were conducted with patients recruited from the center in Richmond, Virginia, and round 3 was conducted with US-Hispanic patients recruited from the center in Texas. All interviews were conducted in person, were audio recorded and transcribed, and lasted approximately 45 minutes. Patient sociodemographic data were obtained from a patient-completed background questionnaire and relevant medical data from patient records. ESM 1 presents additional details about the CD interview methodology.

\subsubsection{Cognitive Debriefing Analyses}

Analysis of the CD interview data was based on a combination of the interview field notes and transcripts. Participants' feedback on the suitability and relevance of the draft NASHCHECK PROM, including issues identified with specific elements (instructions, recall period, response options, and individual items), was collated and summarized to inform the measure's revision.

\section{Results}

\subsection{Literature Review}

The literature review explored the availability of PROMs targeted toward the NASH population. PROMs that have been used in NASH studies include the 36-item Short-Form Health Survey (SF-36) [31], the Chronic Liver Disease Questionnaire (CLDQ) [32], and an NAFLD-specific version of the CLDQ (CLDQ-NAFLD) [33]. The content of the SF-36 and CLDQ are not specific to NASH and may miss issues of importance to NASH. Moreover, while the CLDQNAFLD includes items developed specifically to capture the impacts of NAFLD, neither it nor any other measure to date has followed guidance for the development and validation of a PROM suitable for consideration for regulatory label claims [23, 24].

The review of the published literature identified few published studies $(n=6)$ assessing HRQoL of a biopsyconfirmed NASH population only. Studies presented burden of NAFLD across the spectrum of severity, with NASH sometimes reported as a subgroup; moreover, the terms NASH and NAFLD were not always clearly differentiated. Nonetheless, published literature on the humanistic burden of NASH, although limited, highlights the negative impact that NASH may have on many aspects of patients' lives $[34,35]$. Patients with NASH report significantly increased lifetime rates of depression and anxiety [36], and psychological factors have been found to be linked to weight outcomes for patients with NASH [37-39]. Adults with NASH have lower HRQoL based on the SF-36 than the general population [38]. Further, patients with NASH, relative to those with NAFLD without NASH, report significantly poorer SF-36 physical component scores and significantly poorer scores on the vitality, general health, bodily pain, and role limitations subscales [38].

The narratives from searches of social media reinforce the observation that living with NASH can have a profound impact on many aspects of patients' lives, including their ability to conduct day-to-day activities, their personal and social relationships, and, ultimately, their HRQoL [40, 41]. Clinical experts and patient representatives reviewed the findings from the searches of the literature and social media and confirmed that the findings largely matched their views of the patient experience. Additional areas of impact raised by patient representatives were economic impact (cost of medication, lifestyle management) and patient behavior (motivation and eating habits). Taken together, the results of the reviews and expert input were used to develop an initial conceptual model for NASH.

\subsection{Participant Demographics and Clinical Characteristics}

CE interviews were conducted with 23 patients; 16 had a NASH diagnosis based on biopsy, and seven had a phenotypic diagnosis of NASH. The CD sample included 20 patients overall; 16 had a NASH diagnosis based on biopsy, and four had a phenotypic diagnosis. Characteristics of both $\mathrm{CE}$ and $\mathrm{CD}$ participants included in the analysis are provided in Table 1.

Eight patients who participated in the CE interviews also participated in the CD interviews. The inclusion of some patients in both stages provides further confirmation on the interpretation of concepts emerging from the $\mathrm{CE}$ interview stage and that they were reflected appropriately in the draft NASH-CHECK.

The CE and CD samples were broadly similar in terms of their demographic and clinical characteristics. The most frequently reported comorbidities among all study participants were type 2 diabetes mellitus, hypertension, high cholesterol, and depression.

\subsection{Patient-Reported Impact of NASH}

Analysis of the CE interview data was completed using five sets of transcripts to assess thematic content saturation. Sets 1 through 4 comprised five transcripts each, and the fifth set contained three transcripts. As expected, most themes were identified in the first set of five transcripts analyzed. 
No new symptoms or impacts emerged after the fourth set of transcripts, confirming that concept saturation was reached and supporting that additional interviews would have yielded little or no new concepts of relevance for the construction of a new PRO instrument for NASH.

The symptoms of NASH that emerged from the CE interviews included pain in the upper-right abdomen, fatigue or tiredness, poor sleep quality, and cognitive issues such as impaired memory and reduced focus (Fig. 1). Specifically, in detailing the pain associated with NASH, participants mentioned feelings of discomfort and/or ache, sometimes described as "constant" and "chronic". One participant noted:
"Constant dull ache in my right upper quadrant that radiates to my back."

Participants' description of fatigue included tiredness, exhaustion, and low or no energy levels, even after the completion of normal activities, after a non-strenuous day at work, or after a full night's sleep, noting:

"I get very tired ... normal activities fatigue me."

Participants also described memory issues relating to difficulty retaining and retrieving information, as well as forgetfulness. Poor sleep quality was reported for a range of issues, including frequent awakenings because of the occurrence of pain, difficulty falling asleep, sleeping for short periods of
Table 1 Demographic and disease information: concept elicitation and cognitive debriefing samples

\begin{tabular}{|c|c|c|}
\hline Characteristic & CE sample $(n=23)$ & CD sample $(n=20)$ \\
\hline \multicolumn{3}{|l|}{ Sex } \\
\hline Female & $18(78.3)$ & $11(55.0)$ \\
\hline Male & $5(21.7)$ & $9(45.0)$ \\
\hline Age & $55.9 \pm 10.0(31.0-73.0)$ & $50.6 \pm 10.4(30.0-68.0)$ \\
\hline \multicolumn{3}{|l|}{ Race } \\
\hline White/Caucasian & 19 (82.6) & $13(65.0)$ \\
\hline Black/African American & $3(13.0)$ & $2(10.0)$ \\
\hline Other & $1(4.3)$ & $4(20.0)$ \\
\hline Missing & $0(0.0)$ & $1(5.0)$ \\
\hline \multicolumn{3}{|l|}{ Ethnicity } \\
\hline Hispanic & $1(4.3)$ & $5(25.0)$ \\
\hline Years since diagnosis & $4.1 \pm 2.6$ & $3.9 \pm 3.3$ \\
\hline BMI & $33.5 \pm 5.4(25.8-45.4)$ & $35.9 \pm 6.3(26.2-54.9)$ \\
\hline Obese $^{\mathrm{a}}$ & $16(69.6)$ & $18(90.0)$ \\
\hline \multicolumn{3}{|l|}{ Diagnosis } \\
\hline Biopsy diagnosis & $16(69.6)$ & $16(80.0)$ \\
\hline \multicolumn{3}{|l|}{ Fibrosis grade ${ }^{b}$} \\
\hline F1, perisinusoidal or periportal fibrosis & $1(4.3)$ & $5(25.0)$ \\
\hline F2, perisinusoidal and portal/periportal fibrosis & $5(21.7)$ & $5(25.0)$ \\
\hline $\mathrm{F} 3$, bridging fibrosis & $10(43.5)$ & $6(30.0)$ \\
\hline Phenotypic diagnosis & $7(30.4)$ & $4(20.0)$ \\
\hline \multicolumn{3}{|l|}{ Most frequently reported comorbid health conditions } \\
\hline Type 2 diabetes mellitus & $14(60.9)$ & $14(70.0)$ \\
\hline Hypertension & $16(69.6)$ & $13(65.0)$ \\
\hline Hyperlipidemia & $12(52.2)$ & $11(55.0)$ \\
\hline Depression & $7(30.4)$ & $6(30.0)$ \\
\hline Asthma & $5(21.7)$ & $5(25.0)$ \\
\hline Anxiety & $4(17.4)$ & $4(20.0)$ \\
\hline
\end{tabular}

Data are presented as mean \pm standard deviation (range) or $N(\%)$ unless otherwise indicated $B M I$ body mass index, $C D$ cognitive debriefing, $C E$ concept elicitation

${ }^{a}$ Based on $\mathrm{BMI} \geq 30$

${ }^{\mathrm{b}}$ Based on NASH Clinical Research Network histological scoring system [28] or phenotypic diagnosis (i.e., alanine transaminase levels $\left[\geq 60 \mathrm{IU} / \mathrm{L}\right.$ for males or $\geq 40 \mathrm{IU} / \mathrm{L}$ for females] and BMI $\left[\mathrm{kg} / \mathrm{m}^{2}\right][\geq 23$ in Asian individuals or $\geq 27$ in non-Asian individuals]) and type 2 diabetes mellitus 
time, not having enough sleep, and not wanting to sleep. One participant noted that

"The sharp pain literally would wake me up, like, breathless from a dead sleep. The ... annoying pain is always there."

Almost half of participants also reported the occurrence of itching skin, including the experience of itch on their fingers, hands, elbows, feet, and scalp, noting,

"I do have itching, often."

The broader HRQoL impact of NASH included impaired physical functioning, ability to conduct daily living tasks, reduced quality of relationships, low mood, anxiety, and self-consciousness (Fig. 2). In particular, participants reported difficulty with physical activities, walking, bending, and reaching. According to one participant:

"I used to walk 5 miles a day, and I was riding a bicycle during the summer, too. I can't do any of it now. You know, I just feel like ... you feel like everything's been deprived from you."

Participants also reported difficulty with chores such as sweeping the floor and gardening, with some noting that their ability to complete such tasks had become more difficult. Nearly one-third of the sample reported a negative impact of NASH on relationships, including interactions with family members, particularly children and grandchildren; one participant pointed out,
"You're exhausted; it's hard to be sweet granddaddy when you're worn out and so that limits me from the time."

Participants also reported the impact of NASH on their social activities, restrictions in their ability to dine out or with friends, or feelings of tiredness. The psychological impact of NASH related to emotion and mood, self-confidence, and self-esteem included participants' feelings of stigma related to having "fatty liver disease", the impact on their appearance, and feelings of low mood. Participants noted how they felt judged by others, particularly by those who had no understanding of the condition. More than a quarter of the sample reported the impact of NASH on their mental health, mentioning feeling anxious and depressed,

"It has affected me mentally and physically ... I would

... shed tears and cry to myself."

Finally, participants reported the impact of NASH on their diet, with more than half of the sample making healthy food choices because of NASH and others specifying that their diet had become bland and restrictive.

Figure 3 presents the final conceptual model, which was refined based on the CE interview results and in collaboration with clinical and patient advocacy experts. The $\mathrm{CE}$ interviews confirmed that patients with NASH experience significant impairment in $\mathrm{HRQOL}$ and that patients report aspects of the disease not fully covered by existing PROMs. Refinement of the model informed logical conceptual groupings, including NASH symptoms, activity limitations, and social, psychological, and economic impacts of NASH to be evaluated in planned psychometric analyses.
Fig. 1 Key symptoms reported by concept elicitation sample $(n=23)$

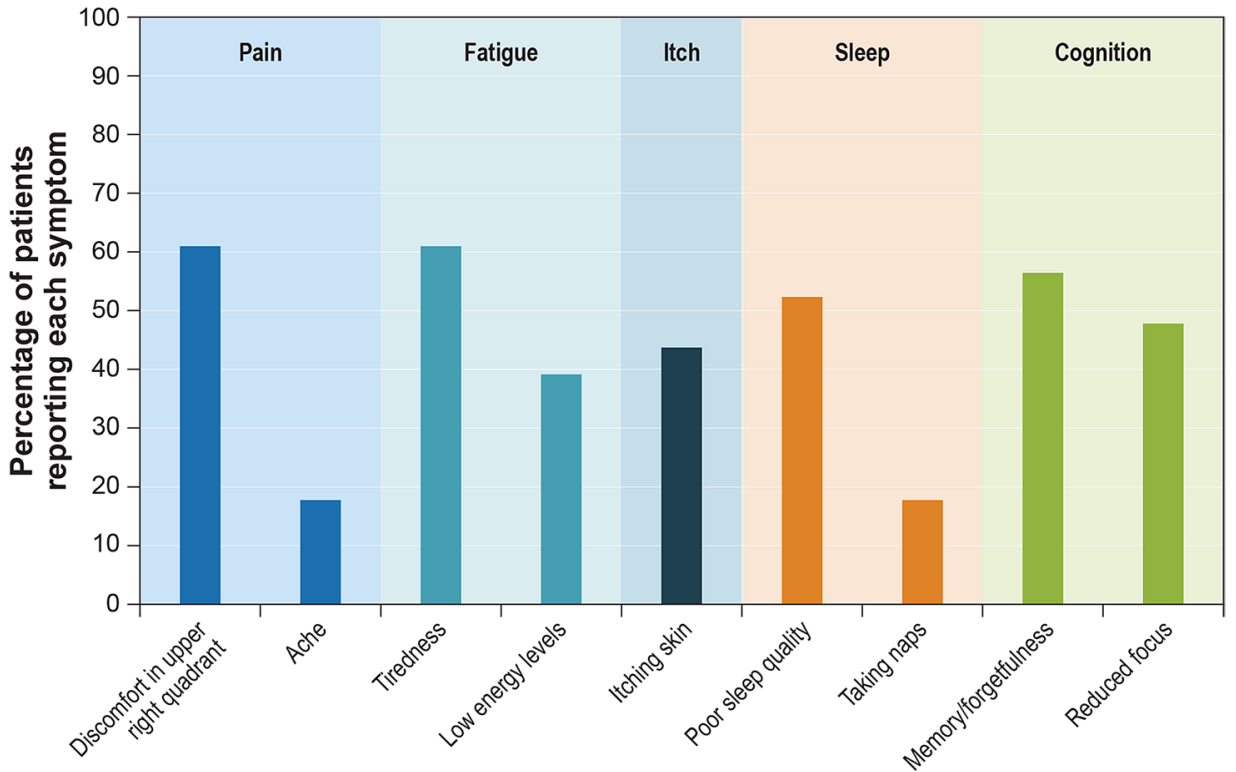

Type of symptom 
Fig. 2 Key health-related quality of life (HRQoL) issues reported by concept elicitation sample $(n=23)$. iADL instrument activities of daily living

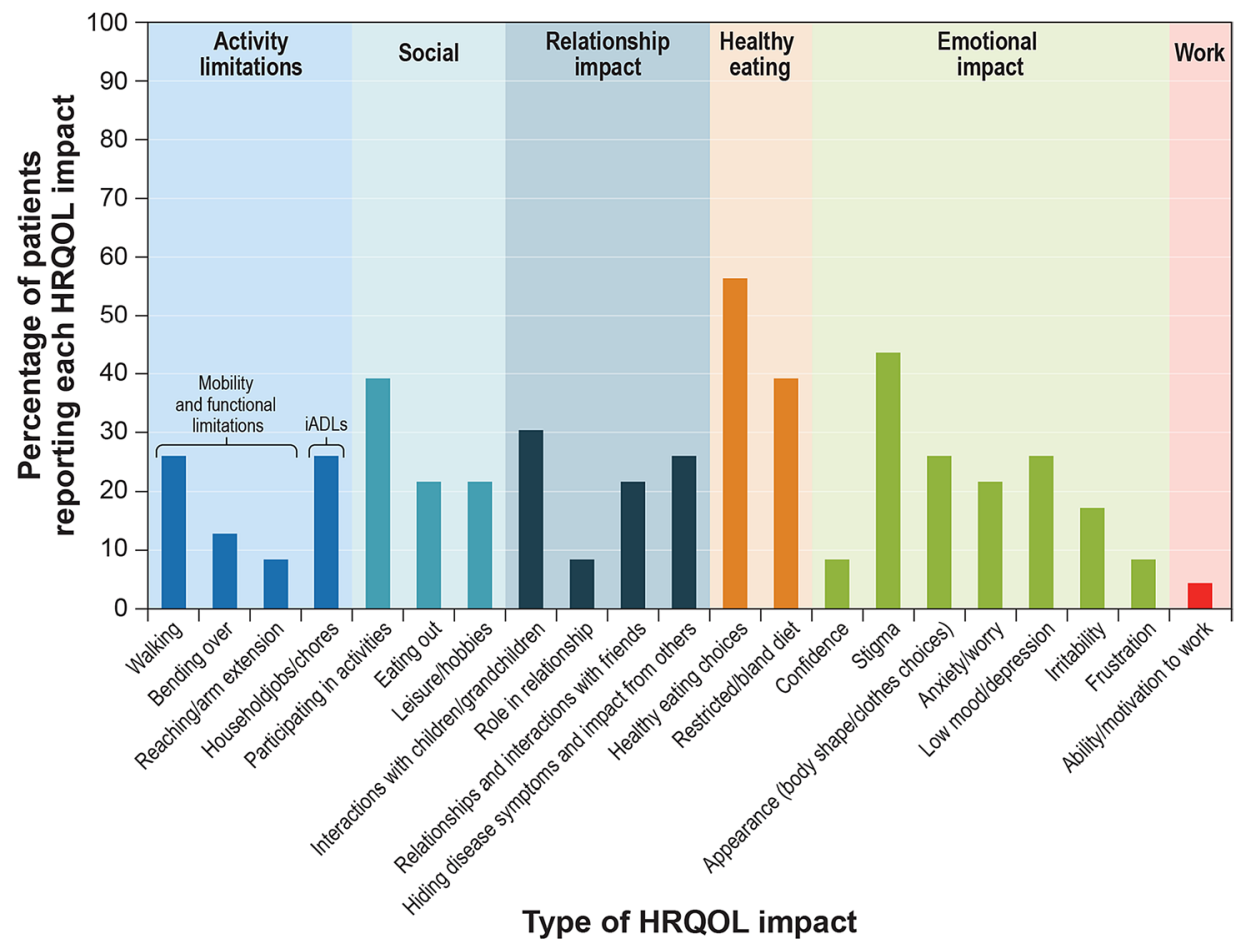

\subsection{NASH-CHECK Development}

The NASH PRO Task Force selected items for the first draft of the US-English NASH-CHECK measure. The first draft of the NASH-CHECK measure comprised 52 items that were representative of the symptoms and HRQoL impacts reported in the qualitative interviews. Overall, 16 symptom items assessing elements of pain, fatigue, itch, sleep disturbance, and cognition and $36 \mathrm{HRQoL}$ items assessing activity limitations, social impact (social activities and relationships), work impact, psychological impact (emotional and self-esteem), and dietary impact were included. A degree of concept duplication was allowed for some of the items in the first draft of the NASH-CHECK so that patient preferences for item phrasing for these items could be evaluated during the first round of $\mathrm{CD}$ interviews. The recall period for the initial item set was 7 days, based on input from clinical experts and patient advocates and the recall periods for PRO measures specific to liver disease, which typically range from 1 to 4 weeks [25]. A 1-week recall was considered appropriate to comprehensively evaluate the symptoms and impacts of NASH while minimizing the potential for recall bias.

Following the first round of $\mathrm{CD}$ interviews, the items considered duplicative or irrelevant by patients were removed, and the total number of items was reduced to 34 (ten symptom, eight activity limitations, and 16 emotion and lifestyle items). The PROM was revised further after the completion of the second round of CD interviews. The number of items was reduced to 31 after three emotion and lifestyle items were removed. In addition, minimal changes were made to instructions and response options. For example, "at its worst" was moved from the instructions to the opening of each symptom item. Patients considered the 1-week recall period to be appropriate, and no changes were made to it. Finally, the last round of $\mathrm{CD}$ interview supported that the NASH-CHECK items were clear, relevant, comprehensive, and easy to complete. No problems were identified in relation to the instructions, recall period, or response format.

The 31-item pilot version of the NASH-CHECK was included in an interventional phase II study and will undergo psychometric analysis to finalize the structure, explore its measurement properties and responsiveness, and inform the interpretation of scores.

\section{Discussion}

This study aimed to explore the patient-perceived impact of NASH and to develop a PROM to evaluate the symptom and HRQoL burden among a real-world sample of patients recruited from clinical practice. A review of the limited published literature on the impact of NASH, consultation with medical and patient advocacy experts, and qualitative CE interviews revealed numerous symptoms and impacts associated with NASH that have a wide impact on patients' dayto-day lives, challenging the notion that NASH is a "silent" disease. Key symptoms reported included pain, particularly 


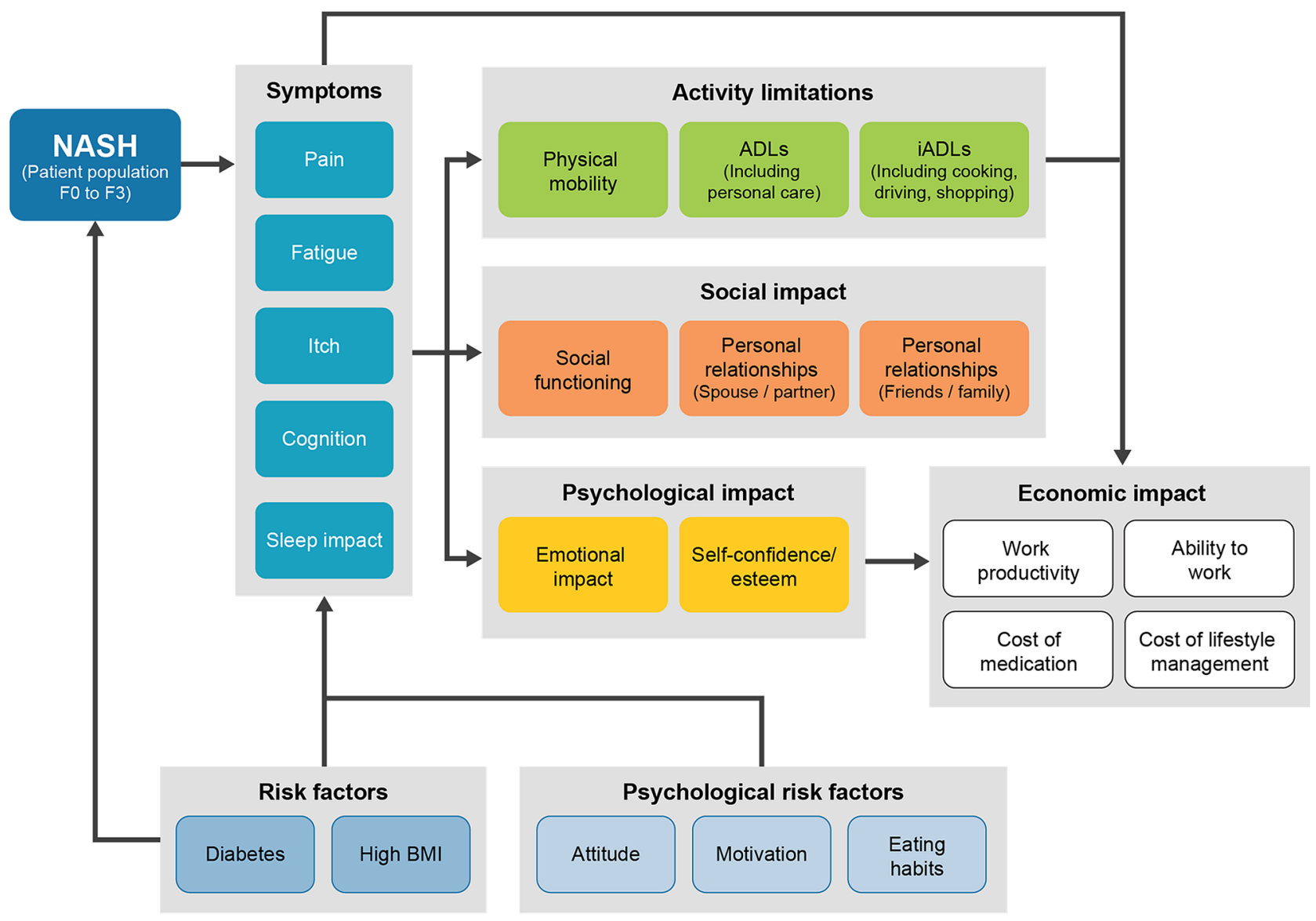

Fig. 3 Final conceptual model for NASH. $A D L$ activity of daily living, $B M I$ body mass index, $i A D L$ instrumental ADL, $N A S H$ non-alcoholic steatohepatitis

in the upper-right quadrant, fatigue, itch, sleep disturbance, and cognitive issues. Key HRQoL impacts included activity limitations, social functioning, psychological issues, work impact, and dietary restrictions. Although patients varied in the number of symptoms and impacts reported, a majority experienced multiple symptoms and HRQoL impacts. Only one patient who had been diagnosed with NASH as part of routine medical screening reported that their NASH was completely asymptomatic with no broader HRQoL impacts experienced.

Development of the NASH-CHECK aligned with regulatory expectations [22-24] and good research practices [40]. The FDA guidance makes a number of recommendations for PROs that are to be used for label claims. These include the development of a conceptual model to guide development activities, clear documentation of the process of item development and refinement, and the evaluation of content validity via qualitative studies to confirm that the items and domains covered by a PRO are appropriate and comprehensive. The development activities of the NASH-CHECK were guided by a clear conceptual model that was refined during the development process. Input from patients with NASH was central to development of the NASH-CHECK content. Items for the measure were derived from qualitative interviews, during which content saturation was achieved, and $\mathrm{CD}$ interviews were used to ensure the suitability of the measure for patients with NASH. Specifically, in the CD interviews, participants reported the NASH-CHECK measure to be relevant, acceptable, understandable, and clear. The instructions, response options, and recall period were considered appropriate, and the recall period is consistent with recommendations from the FDA guidance against lengthy recall periods [23]. Minor changes were made to some instructions and item wording to improve clarity. The structure and concepts measured by the NASH-CHECK measure were maintained during refinement of the measure. The final NASH-CHECK measure was reduced to 31 items (ten symptom items and $21 \mathrm{HRQoL}$ items) based on patient preferences for item relevance, acceptability, and comprehensibility.

Findings from this study also will support future patient-centered research initiatives in NASH. The use of 
NASH-CHECK with patients will improve understanding of the humanistic burden associated with NASH. Additional analyses are planned to finalize the dimensional structure and explore the psychometric properties of the NASH-CHECK.

Some limitations of this study must be considered. The participants in the $\mathrm{CE}$ and $\mathrm{CD}$ interviews were sampled from two clinical sites in the USA. A non-probability sampling approach was used, which has the potential to introduce bias because the sample may not be reflective of the wider population. Participation in the interviews was entirely voluntary, and it is possible that such self-selection could also lead to selection bias. Nevertheless, results from preliminary qualitative research conducted with a geographically diverse sample of 35 patients with NASH in North America, South America, Europe, Asia, and Australia are aligned with our findings, in that patients reported similar impacts of NASH $[14,42]$. In addition, ten CE interview participants and six CD interview participants had grade F3 fibrosis, at the upper end of the severity range of non-cirrhotic NASH. We considered that patients with advanced fibrosis were more likely to have symptoms, and it was important that the sample included a sufficient proportion of these patients to adequately capture their experiences with NASH. Finally, while most participants had biopsy-confirmed NASH, some participants had phenotypic diagnosis (seven CE interview participants and four CD interview participants), which, in the absence of a clear gold standard for clinical biomarkers for NASH, is generally accepted by the clinical community. These patients were recruited from a specialty center, conferring reasonable confidence in their NASH diagnosis.

\section{Conclusion}

This study revealed key areas of the symptomatic and broader HRQoL impacts of NASH, informing the development and establishing the content validity of the NASHCHECK. The NASH-CHECK is a disease-specific PROM assessing symptoms and HRQoL that have been found to be relevant, comprehensive, and acceptable to patients and is suitable for use in both clinical trials and routine practice. Future analyses are planned to explore its psychometric properties and validate the measure for additional populations, including individuals with cirrhotic NASH.

Acknowledgements This research was conducted by the NASH PRO Task Force, an international interdisciplinary collaborative group. The authors thank Andrew Langford of the British Liver Trust (UK), Neetu Agashivala of Novartis Pharmaceuticals, Inc. (USA), T. Michelle Brown of RTI Health Solutions (USA), Rebecca Collen of Virginia Commonwealth University (USA), and Eric Lawitz of the Texas Liver Institute (USA) for their contributions to the study. We also thank the patients for their contributions to the research and Kate Lothman from
RTI Health Solutions (USA) for her medical writing support in preparing this manuscript. Medical writing services were funded by Novartis Pharma AG.

\section{Declarations}

Funding This study and preparation of this publication were funded by Novartis Pharma AG.

Conflict of interest This study was completed under a research contract between RTI Health Solutions and Novartis Pharma AG and was funded by Novartis Pharma AG. LCD, JT, and CS are salaried employees of RTI Health Solutions. MMB and CAB are salaried employees of Novartis. DC is a salaried employee of the Global Liver Institute, which received consulting fees from Novartis in support of this research. AJS has received research grants and consulting fees from Novartis, which funded this study. QMA has received research grants and consulting fees from Novartis and is participating in research collaborations with Novartis, which funded this study.

Availability of data and material To protect participant confidentiality, the data set for this study cannot be shared.

Ethics approval This study was conducted in accordance with the ethical standards of the Western Institutional Review Board (Puyallup, Washington) and with the 1964 Helsinki declaration and its later amendments or comparable ethical standards.

Consent All participants interviewed in this study provided informed consent.

Author contributions MMB conceived of the study, contributed to the study design, secured funding, interpreted the data, and critically revised the manuscript for important intellectual content. LCD designed the study; acquired, analyzed, and interpreted the data; provided study supervision; and critically revised the manuscript for important intellectual content. JT and CS contributed to the study design; acquired, analyzed, and interpreted the data; and critically revised the manuscript for important intellectual content. DC, CAB, QMA, and AJS provided clinical and other insights on the study design and study concepts, interpreted the data, and critically revised the manuscript for important intellectual content.

Open Access This article is licensed under a Creative Commons Attribution-NonCommercial 4.0 International License, which permits any non-commercial use, sharing, adaptation, distribution and reproduction in any medium or format, as long as you give appropriate credit to the original author(s) and the source, provide a link to the Creative Commons licence, and indicate if changes were made. The images or other third party material in this article are included in the article's Creative Commons licence, unless indicated otherwise in a credit line to the material. If material is not included in the article's Creative Commons licence and your intended use is not permitted by statutory regulation or exceeds the permitted use, you will need to obtain permission directly from the copyright holder. To view a copy of this licence, visit http://creativecommons.org/licenses/by-nc/4.0/.

\section{References}

1. Bellentani S, Marino M. Epidemiology and natural history of nonalcoholic fatty liver disease (NAFLD). Ann Hepatol. 2009;8(suppl 1):S4-8. 
2. Alkhouri N, McCullough AJ. Noninvasive diagnosis of NASH and liver fibrosis within the spectrum of NAFLD. Gastroenterol Hepatol. 2012;8(10):661-8.

3. Sanyal AJ, Chalasani N, Kowdley KV, et al. Pioglitazone, vitamin e, or placebo for nonalcoholic steatohepatitis. N Engl J Med. 2010;362(18):1675-85.

4. Vernon G, Baranova A, Younossi ZM. Systematic review: the epidemiology and natural history of non-alcoholic fatty liver disease and non-alcoholic steatohepatitis in adults. Aliment Pharmacol Ther. 2011;34(3):274-85.

5. Wanless IR, Lentz JS. Fatty liver hepatitis (steatohepatitis) and obesity: an autopsy study with analysis of risk factors. Hepatology. 1990;12:1106-10.

6. McCullough AJ. Pathophysiology of nonalcoholic steatohepatitis. J Clin Gastroenterol. 2006;40:S17-29.

7. Brunt EM, Neuschwander-Tetri BA, Oliver D, Wehmeier KR, Bacon BR. Nonalcoholic steatohepatitis: histologic features and clinical correlations with 30 blinded biopsy specimens. Hum Pathol. 2004;35:1070-82.

8. Chalasani N, Younossi Z, Lavine JE, et al. The diagnosis and management of non-alcoholic fatty liver disease: practice guideline by the American Association for the Study of Liver Diseases, American College of Gastroenterology, and the American Gastroenterological Association. Hepatology. 2012;55(6):2005-23.

9. Powell EE, Cooksley WG, Hanson R, et al. The natural history of nonalcoholic steatohepatitis: a follow-up study of forty-two patients for up to 21 years. Hepatology. 1990;11(74):80.

10. Jansen PL. Non-alcoholic steatohepatitis. Eur J Gastroenterol Hepatol. 2004;16(11):1079-85.

11. Heeringa M, Hastings A, Yamazaki S, et al. Serum biomarkers in nonalcoholic steatohepatitis: value for assessing drug effects? Biomark Med. 2012;6:743-57.

12. Khedmat H, Taheri S. Non-alcoholic steatohepatitis: an update in pathophysiology, diagnosis and therapy. Hepat Mon. 2011;11:74-85.

13. Balp MM, Krieger N, Przybysz R, et al. The burden of nonalcoholic steatohepatitis (NASH) among patients from Europe: a real-world patient-reported outcomes study. JHEP Rep. 2019;1:151-64.

14. Cook NS, Nagar SH, Jain A, et al. Understanding patient preferences and unmet needs in non-alcoholic steatohepatitis (NASH): insights from a qualitative online bulletin board study. Adv Ther. 2019;36(2):478-91.

15. Assimakopoulos K, Karaivazoglou K, Tsermpini EE, Diamantopoulou G, Triantos C. Quality of life in patients with nonalcoholic fatty liver disease: a systematic review. J Psychosom Res. 2018;112:73-80.

16. Younossi ZM, Henry L. Economic and quality-of-life implications of non-alcoholic fatty liver disease. Pharmacoeconomics. 2015;33(12):1245-53.

17. Huber Y, Boyle M, Hallsworth K, et al. Health-related quality of life in nonalcoholic fatty liver disease associates with hepatic inflammation. Clin Gastroenterol Hepatol. 2019;17:2085-92.

18. Loomba R, Lutchman G, Kleiner DE, et al. Clinical trial: pilot study of metformin for the treatment of non-alcoholic steatohepatitis. Aliment Pharmacol Ther. 2009;29:172-82.

19. Ratziu V, Sheikh MY, Sanyal AJ, et al. A phase 2, randomized, double-blind, placebo-controlled study of GS-9450 in subjects with nonalcoholic steatohepatitis. Hepatology. 2012;55:419-28.

20. Zein CO, Yerian LM, Gogate P, et al. Pentoxifylline improves nonalcoholic steatohepatitis: a randomized placebo-controlled trial. Hepatology. 2011;54:1610-9.

21. Doward LC, Gnanasakthy A, Baker MG. Patient reported outcomes: looking beyond the label claim. Health Qual Life Outcomes. 2010;8:89.
22. US Food and Drug Administration (US FDA). CDER patientfocused drug development. July 20, 2020. https://www.fda.gov/ drugs/development-approval-process-drugs/cder-patient-focus ed-drug-development. Accessed 09 Aug 2020.

23. US Food and Drug Administration (US FDA). Guidance for industry. patient-reported outcome measures: use in medical product development to support labeling claims. Dec 2009. https://www. fda.gov/regulatory-information/search-fda-guidance-documents/ patient-reported-outcome-measures-use-medical-product-devel opment-support-labeling-claims. Accessed 24 May 2019.

24. European Medicines Agency. Reflection paper on the regulatory guidance for the use of health-related quality of life (HRQL) measures in the evaluation of medicinal products. Committee for Medicinal Products for Human Use. 27 July 2005. https://www. ema.europa.eu/en/documents/scientific-guideline/reflection-paper -regulatory-guidance-use-healthrelated-quality-life-hrql-measu res-evaluation_en.pdf. Accessed 24 May 2019.

25. McSweeney L, Breckons M, Fattakhova G, et al. Health-related quality of life and patient-reported outcome measures in NASHrelated cirrhosis. JHEP Rep. 2020;2(3):100099.

26. British Liver Trust. 2015. https://www.britishlivertrust.org.uk/. Accessed 01 Sept 2015.

27. Global Liver Institute. 2015. https://www.globalliver.org/. Accessed 01 Sept 2015.

28. Guest G, Bunce A, Johnson L. How many interviews are enough? An experiment with data saturation and variability. Field Methods. 2006;18:59-82.

29. Kleiner DE, Brunt EM, Van Natta M, et al. Nonalcoholic Steatohepatitis Clinical Research Network. Design and validation of a histological scoring system for nonalcoholic fatty liver disease. Hepatology. 2005;41:1313-21.

30. Braun V, Clarke V. Using thematic analysis in psychology. Qual Res Psychol. 2006;3:77-101.

31. Ware JE Jr, Sherbourne CD. The MOS 36-item short-form health survey (SF-36) I Conceptual framework and item selection. Med Care. 1992;30:473-83.

32. Younossi ZM, Guyatt G, Kiwi M, et al. Development of a disease specific questionnaire to measure health related quality of life in patients with chronic liver disease. Gut. 1999;45:295-300.

33. Younossi ZM, Stepanova M, Henry L, et al. A disease-specific quality of life instrument for non-alcoholic fatty liver disease and non-alcoholic steatohepatitis: CLDQ-NAFLD. Liver Int. 2017;37:1209-18.

34. Doward LC, Balp M-M, Twiss J, et al. Understanding the patientperceived impact of nonalcoholic steatohepatitis: raising the volume on a silent disease. Hepatology. 2017;66:1182A.

35. Newton JL, Jones DE, Henderson E, et al. Fatigue in non-alcoholic fatty liver disease (NAFLD) is significant and associates with inactivity and excessive daytime sleepiness but not with liver disease severity or insulin resistance. Gut. 2008;57:807-13.

36. Elwing JE, Lustman PJ, Wang HL, et al. Depression, anxiety, and nonalcoholic steatohepatitis. Psychosomatic Med. 2006;68:563-9.

37. Stewart KE, Haller DL, Sargeant C, et al. Readiness for behaviour change in non-alcoholic fatty liver disease: implications for multidisciplinary care models. Liver Int. 2015;35:936-43.

38. David K, Kowdley KV, Unalp A, et al. Quality of life in adults with nonalcoholic fatty liver disease: baseline data from the Nonalcoholic Steatohepatitis Clinical Research Network. Hepatology. 2009;49:1904-12.

39. Tapper EB, Lai M. Weight loss results in significant improvements in quality of life for patients with nonalcoholic fatty liver disease: a prospective cohort study. Hepatology. 2016;63(4):1184-9. https ://doi.org/10.1002/hep.28416.

40. Harrington RL, Hanna ML, Oehrlein EM, et al. Defining patient engagement in research: results of a systematic review and 
analysis: report of the ISPOR Patient-Centered Special Interest Group. Value Health. 2020;23(6):677-88.

41. Doward LC, Balp M-M, Stewart KE, et al. Exploring the patient perceived impact of non-alcoholic steatohepatitis. J Hepatol. 2017;66:S422-3.
42. Palsgrove AC, Hazra S, Ferguson B, Cheng R, Cole JC. Development of a conceptual framework for assessing disease-specific patient-reported outcomes in nonalcoholic steatohepatitis. Value Health. 2016;19:A94.

\section{Affiliations}

\section{Lynda C. Doward ${ }^{1} \cdot$ Maria-Magdalena Balp $^{2}$ - James Twiss ${ }^{1} \cdot$ Christina Slota $^{3} \cdot$ Donna Cryer $^{4} \cdot$ Clifford A. Brass $^{5}$. Quentin M. Anstee ${ }^{6,7} \cdot$ Arun J. Sanyal $^{8}$}

Maria-Magdalena Balp

maria-magdalena.balp@novartis.com

James Twiss

jtwiss@ rti.org

Christina Slota

chrisslota@rti.org

Donna Cryer

dcryer@globalliver.org

Clifford A. Brass

clifford.brass@novartis.com

Quentin M. Anstee

quentin.anstee@newcastle.ac.uk

Arun J. Sanyal

arun.sanyal@vcuhealth.org
1 RTI Health Solutions, The Pavilion, Towers Business Park, Wilmslow Road, Didsbury, Manchester M20 2LS, UK

2 Novartis Pharma AG, Basel, Switzerland

3 RTI Health Solutions, Research Triangle Park, NC, USA

4 Global Liver Institute, Washington, DC, USA

5 Novartis Pharmaceuticals, Inc., East Hanover, NJ, USA

6 Translational and Clinical Research Institute, The Medical School, Newcastle University, Framlington Place, Newcastle upon Tyne, UK

7 Newcastle NIHR Biomedical Research Centre, Newcastle upon Tyne Hospitals NHS Foundation Trust, Newcastle upon Tyne, UK

8 Virginia Commonwealth University, Richmond, USA 